\title{
Obstructions for steady-state controllability
}

\author{
Yacine Chitour \\ Supélec \\ Plateau du Moulon \\ 3, rue Joliot-Curie \\ 91192 Gif-sur-Yvette cedex (France) \\ E-mail: Yacine.Chitourdss.supelec.fr \\ Jean-Michel Coron \\ Laboratoire de mathématiques \\ Université Paris-Sud - Bât 425 \\ 91405 Orsay Cedex (France) \\ E-mail: Jean-Michel.Coron@math.u-psud.fr

\section{Mauro Garavello*} \\ Dipartimento di Matematica e Applicazioni, \\ Università di Milano Bicocca \\ Via R. Cozzi 53 - Edificio U5 \\ 20125 Milano (Italy) \\ E-mail: mauro.garavello@unimib.it
}

In this paper, we investigate the connections between controllability properties of distributed systems and existence of non zero entire functions subject to restrictions on their growth and on their sets of zeros. Exploiting these connections, we first show that, for generic bounded open domains in dimension $n \geq 2$, the steady-state controllability for the heat equation, with boundary controls dependent only on time, does not hold. In a second step, we study a model of water tank whose dynamics is given by a wave equation on a two-dimensional bounded open domain. We provide an obstruction for the steady-state controllability of such a system, where the control acts on the boundary and is only dependent on time, and using that obstruction, prove that the steady-state controllability does not hold for generic tank shapes.

Control Systems: Theory, Numerics and Applications

30 March - 1 April 2005

Rome

${ }^{*}$ Speaker. 


\section{Inotroduction}

We consider the steady-state controllability in finite time for control systems given by some partial differential equations. In this paper (and as it will be clear from the statements of the results), "steady-state" refers to independence with respect to the state variable, i.e. steady-states are simply constant functions (of the state variable). Moreover, the control strategies considered here are only time dependent. For certain control systems modeled by a partial differential equation, we investigate whether, given two arbitrary steady states of the control system, one can steer the first steady state to the second one in finite time by means of a suitable (only time-dependent) control. We refer to such property as the steady-state controllability for the corresponding control system. That class of problems has been introduced by N. Petit and P. Rouchon in [19] for a control system modeling a water tank. The control problem they addressed consists of steering in finite time the tank from one steady state to another one, using as a control the acceleration of the tank (this leads to two boundary controls which are only dependent on time). The dynamics is given by a linear wave equation on a bounded open set of $\mathbb{R}^{2}$, as detailed below. They solved positively the problem in the case where the tank is either a disc or a rectangle. For more general tank shapes, they asserted that the problem of steady-state controllability is open. In the same spirit, P. Rouchon, in [20], considered the steady-state controllability for the heat equation on an open, bounded and non empty subset $\Omega$ of $\mathbb{R}^{n}$, where the control is only dependent on time and acts on the boundary, i.e. $y(t, x)=u(t)$ on $\partial \Omega$ with the usual notations. It is well-known (see [ [ Theorem IV.2.7, page 187] and [6, Theorem 2.2]) that there is a negative answer to the null controllability in finite time of this control system, that is there are states which cannot be steered to zero in finite time. Moreover, stronger negative results, showing that, in fact, very few states can be steered to zero in finite time for the heat equation were obtained by S. Micu and E. Zuazua in [13, 14] for the case where the domain $\Omega$ is a half-space (see also [15] for a fractional order parabolic equation). In [20], P. Rouchon raised the following question: is it possible to steer the special initial data $y_{0} \equiv 1$ to zero in finite time? We use $(R)$ to denote that particular control problem. P. Rouchon shows that $(R)$ has a solution if $n=1$ or if $\Omega$ is a ball in $\mathbb{R}^{n}$ and asks what is the answer for general open subsets in $\mathbb{R}^{n}$ with $n \geqslant 2$.

The first result of the present paper is the characterization of a property on $\Omega$, denoted $(A)$, which is an obstruction to the steady-state controllability for the heat equation with boundary controls that depend only on time. Property $(A)$ is expressed in terms of the averages on $\Omega$ of the eigenfunctions of the Laplace-Dirichlet operator. We show that property $(A)$ holds for generic open subsets $\Omega \in \mathbb{R}^{n}, n \geq 2$, of class $C^{3}$. Therefore, for generic domains $\Omega$, question $(R)$ has a negative answer. Finally, in the case where $\Omega$ is a parallelepiped, we show that, again, property $(A)$ holds, and thus, even if the domain is not of class $C^{3}, y_{0} \equiv 1$ cannot be steered to zero in finite time.

The second result concerns the control problem for the water tank. We again characterize a property $(B)$ on the shape of the tank, expressed in terms of averages on the boundary of the tank of the eigenfunctions of the Laplace-Neumann operator, which turns out to be an obstruction to the steady-state controllability of the associated control system. The shape of a tank is an open, bounded, connected and non empty subset $\Omega$ of $\mathbb{R}^{2}$. We also show that property $(B)$ holds for generic tank shapes of class $C^{3}$.

The strategy we adopt consists in performing a Laplace transform with respect to the time $t$. 
The steady-state controllability issue in time $T>0$ for both control systems is now translated into a problem of complex analysis, namely, the existence of a non-zero holomorphic function $f: \mathbb{C} \rightarrow \mathbb{C}$ such that:

(a) $|f(s)| \leq C_{0} e^{T \max \{0, \operatorname{Re}(s)\}}$;

(b) For every distinct eigenvalue $\lambda_{i}$ of $-\Delta$, either $f\left(\lambda_{i}\right)=0$ (for the control of the heat equation) or $f\left(\lambda_{i}\right)$ belongs to a special one dimensional linear subspace of $\mathbb{C}^{2}$ (for the water tank control system).

Here, $C_{0}$ is a positive constant independent of $s \in \mathbb{C}$ and $-\Delta$ denotes either the Laplace-Dirichlet or the Laplace-Neumann operator. Condition $(a)$ is a consequence of the fact that controllability must be achieved in finite time and, thus, it is a simply result of the Paley-Wiener theorem. Condition $(b)$ is the "infinite-dimensional" version of a standard fact of linear system theory: $\lambda_{i} \in \sigma(-\Delta)$ is a pole of the transfer function associated to the control system under consideration, which is (almost) nothing else but the resolvent of $-\Delta$. For these two control systems, it turns out that null-controllability in finite time would imply the existence of a non-zero entire function subject to restrictions so strong that only the zero function would fulfill them. A contradiction is reached, and so the conclusions.

Before closing the introduction, let us make one remark. It would be interesting to use the link between controllability and complex analysis to derive a constructive way for actual synthesis of the motion. Indeed, as described below, it amounts solving an interpolation problem in a restricted class of entire functions.

\section{Heat equation}

Let $\Omega$ be an open, bounded and non empty subset of $\mathbb{R}^{n}$, with $n \geqslant 2$. For $y_{0} \in L^{2}(\Omega)$ and $T>0$, consider the heat equation

$$
\begin{cases}y_{t}(t, x)-\Delta y(t, x)=0, & \text { if }(t, x) \in(0, T) \times \Omega, \\ y(0, x)=y_{0}, & \text { if } x \in \Omega, \\ y(t, x)=u(t), & \text { if } x \in \partial \Omega\end{cases}
$$

where $u \in L^{2}(0, T)$ is the control. Let us first recall classical results about weak solutions to the Cauchy problem (2.1). Let $y_{0} \in L^{2}(\Omega), T>0$ and $u \in L^{2}(0, T)$. A weak solution to the Cauchy problem (2.1) is a function $y \in C^{0}\left([0, T] ; L^{2}(\Omega)\right)$ such that, for every $\tau \in[0, T]$ and every $\theta \in$ $C^{1}\left([0, T] ; L^{2}(\Omega)\right) \cap C^{0}\left([0, T] ; H_{0}^{1}(\Omega)\right)$ with

$$
\theta_{t}+\Delta \theta=0 \text { in } C^{0}\left([0, T] ; H^{-1}(\Omega)\right)
$$

one has

$$
\begin{gathered}
\int_{\Omega} y(\tau, x) \theta(\tau, x) d x-\int_{\Omega} y_{0}(x) \theta(0, x) d x= \\
\int_{0}^{\tau} u(t)\left(\int_{\Omega} \theta_{t}(t, x) d x\right) d t .
\end{gathered}
$$


Of course, every $y \in C^{1}\left([0, T] ; L^{2}(\Omega)\right) \cap C^{0}\left([0, T] ; H^{1}(\Omega)\right)$, which is a classical solution to (2.1) is a weak solution to (2.1). It is also well known that, for every $y_{0} \in L^{2}(\Omega), T>0$ and $u \in L^{2}(0, T)$, there exists one and only one weak solution $y$ to (2.1). That unique $y$ is called the solution to the Cauchy problem (2.1).

The problem of null controllability associated to (2.1) goes as follows. Given $y_{0} \in L^{2}(\Omega)$, does there exist $T>0$ and $u \in L^{2}(0, T)$ such that the solution of the Cauchy problem (2.1) satisfies $y(T, \cdot)=0$ ? The answer to that question is negative, as shown by H. Fattorini in [6. Theorem 2.2] and by S. Avdonin and S. Ivanov in [2, Theorem IV.2.7, page 187]; see also the papers [13, 14, 15] by S. Micu and E. Zuazua, for even stronger negative results for similar questions.

In this section, we look at a particular $y_{0}$, namely $y_{0} \equiv 1$, and want to see if it is possible to steer that special $y_{0}$ to 0 in finite time, that is, again, does there exists $T>0$ and a control $u \in L^{2}(0, T)$ such that the solution $y$ to the Cauchy problem (2.1) satisfies $y(T, \cdot)=0$ ? Of course, a positive answer to that question is equivalent to the steady-state controllability, i.e. given two constant functions $y_{0} \equiv C_{0}, y_{1} \equiv C_{1}$, does there exist $T>0$ and $u \in L^{2}(0, T)$ such that the solution $y$ to (2.1) satisfies $y(0, \cdot)=y_{0}$ and $y(T, \cdot)=y_{1}$ ? As mentioned in the introduction, P. Rouchon showed in [20] that the steady-state controllability holds for $n=1$ or if $\Omega$ is a ball in $\mathbb{R}^{n}$ and asks what is the answer for general open subsets of $\mathbb{R}^{n}, n \geqslant 2$.

We use $-\Delta_{\Omega}^{D}$ to denote the Laplace-Dirichlet operator defined next,

$$
\begin{aligned}
\mathcal{D}\left(-\Delta_{\Omega}^{D}\right) & :=\left\{v \in H_{0}^{1}(\Omega) ; \Delta v \in L^{2}(\Omega)\right\}, \\
-\Delta_{\Omega}^{D} v & :=-\Delta v, \forall v \in \mathcal{D}\left(-\Delta_{\Omega}^{D}\right) .
\end{aligned}
$$

Let us introduce the definition of Property $(A)$, which turns out to be an obstruction for steering $y_{0} \equiv 1$ to 0 in finite time.

Definition 1. The open set $\Omega$ has the property $(A)$ if there exists a sequence $\left(r_{k}\right)_{k \in \mathbb{N}^{*}}$ of distinct eigenvalues of $-\Delta_{\Omega}^{D}$ such that

(i) one has

$$
\sum_{k=1}^{\infty} \frac{1}{r_{k}}=\infty
$$

(ii) for every $k \in \mathbb{N}^{*}$, there exists an eigenfunction $w$ of the operator $-\Delta_{\Omega}^{D}$ corresponding to the eigenvalue $r_{k}$ such that

$$
\int_{\Omega} w d x \neq 0
$$

We are now able to state the main results of this section.

Theorem 1. Let $\Omega$ be a bounded, open and non empty subset of $\mathbb{R}^{n}, n \geqslant 2$. If $\Omega$ has the property (A), then one cannot steer $y_{0} \equiv 1$ to 0 in finite time.

Proof. Assume that property (A) holds for a bounded, open and non empty subset $\Omega \subset \mathbb{R}^{n}$, $n \geq 2$. We suppose by contradiction that there exist $T>0$ and $u \in L^{2}(0, T)$ such that the solution $y$ to the Cauchy problem (2.1) with

$$
y_{0} \equiv 1
$$


satisfies

$$
y(T, \cdot)=0 .
$$

Let $\lambda$ be an eigenvalue of $-\Delta_{\Omega}^{D}$ and $w$ be an eigenfunction associated to $\lambda$. Consider the function $\theta \in C^{\infty}\left([0, T] ; H_{0}^{1}(\Omega)\right)$ defined by

$$
\theta(t, x):=e^{\lambda t} w(x) .
$$

Then $\theta$ satisfies (2.2). Hence, using (2.3) with $\tau:=T$, (2.6) and (2.7), one gets

$$
B(\lambda) \int_{\Omega} w d x=0
$$

where $B: \mathbb{C} \rightarrow \mathbb{C}$ is defined by

$$
B(s):=1+s \int_{0}^{T} u(t) e^{s t} d t .
$$

Since property (A) holds for $\Omega$, it results that $B$ vanishes on a sequence $\left(r_{k}\right)_{k \in \mathbb{N}^{*}}$ of distinct positive real numbers satisfying (2.4). By the easy part of the Paley-Wiener theorem, the function $B$ is holomorphic on $\mathbb{C}$ and there exists $C>0$ such that

$$
|B(s)| \leqslant C e^{T \max \{0, R e(s)\}}, \forall s \in \mathbb{C} .
$$

We then apply the following lemma.

Lemma 1. Let $f: \mathbb{C} \rightarrow \mathbb{C}$ be a holomorphic function such that, for some $C>0$,

$$
|f(s)| \leqslant C e^{C|R e(s)|}, \forall s \in \mathbb{C} .
$$

Let us assume that there exists a sequence $\left(r_{k}\right)_{k \geqslant 1}$ of distinct positive real numbers such that (2.4) holds and

$$
f\left(r_{k}\right)=0, \forall k \geqslant 1 \text {. }
$$

Then, $f$ is identically equal to 0 .

Lemma 11 is a consequence of a much more general theorem due to Cartwright and Levinson; see [10, Theorem 1, p. 127]. Applying Lemma 11 with $f:=B$, we conclude that $B$ is identically equal to zero. This contradicts the fact that $B(0)=1$. Theorem 1 is proved.

We use here notations and results of [1, ㄱ, 24]. Let $\mathcal{R}\left(\mathbb{R}^{n}\right)$ be the set of all non empty bounded open subsets $\Omega$ of class $C^{3}$. To state the result, one needs to define a topology on $\mathcal{R}\left(\mathbb{R}^{n}\right)$. We follow a construction closely related to that proposed by R. Hamilton in [7] pages 86-87]. For $\Omega \in \mathcal{R}\left(\mathbb{R}^{n}\right)$, let $\xi \in C^{3}\left(\partial \Omega ; \mathbb{R}^{n}\right)$ be such that

$$
\xi(x) \cdot v(x)>0, \forall x \in \partial \Omega
$$

where $v \in C^{2}\left(\partial \Omega, \mathbb{R}^{n}\right)$ denotes the outward normal to $\Omega$.

Let $\varepsilon_{0}>0$ be small enough so that the two following properties hold.

(i) For every $x$ in $\mathbb{R}^{n}$ such that dist $(x, \partial \Omega)<\varepsilon_{0}$, there exists a unique $\pi(x) \in \partial \Omega$ such that $x-\pi(x)$ is parallel to $\xi(\pi(x))$. 
(ii) The map $x \mapsto \pi(x)$ is of class $C^{3}$ on the open set $\left\{x \in \mathbb{R}^{n}\right.$; $\left.\operatorname{dist}(x, \partial \Omega)<\varepsilon_{0}\right\}$.

Let $\varepsilon>0$ and $\eta \in C^{3}(\partial \Omega)$ be such that

$$
|\eta|_{C^{3}(\partial \Omega)}<\varepsilon
$$

Define

$$
\begin{aligned}
\Omega_{\eta}:=\left\{x \in \Omega ; \operatorname{dist}(x, \partial \Omega) \geqslant \varepsilon_{0}\right\} \cup\left\{x \in \mathbb{R}^{n} ;\right. \\
\left.\quad \operatorname{dist}(x, \partial \Omega)<\varepsilon_{0} \text { and }(x-\pi(x)) \cdot \xi(\pi(x))<\eta(\pi(x))\right\} .
\end{aligned}
$$

There exists $\varepsilon_{1}>0$ such that, for every $\eta \in C^{3}(\partial \Omega)$ with $|\eta|_{C^{3}(\partial \Omega)}<\varepsilon_{1}, \Omega_{\eta}$ is a bounded subset of $\mathbb{R}^{n}$ of class $C^{3}$. Let $\mathcal{V}(\varepsilon)$ be the set of all the $\Omega_{\eta}$ with $\eta \in C^{3}(\partial \Omega)$ satisfying (2.13). We define a topology on $\mathcal{R}\left(\mathbb{R}^{n}\right)$ by considering the sets $\mathcal{V}(\varepsilon)$, with $\varepsilon \in\left(0, \varepsilon_{1}\right)$, as a base of neighborhoods of $\Omega$, i.e. every neighborhood of $\Omega$ in $\mathcal{R}\left(\mathbb{R}^{n}\right)$ contains some $\mathcal{V}(\varepsilon)$ for $\varepsilon \in\left(0, \varepsilon_{1}\right)$ small enough. (One easily checks that this topology is independent of the choice of $\xi$ and $\varepsilon_{1}$.) Recall that a topological space is a Baire space if any residual set, i.e. any intersection of denumerable open dense subsets, is dense. Since, for every $\Omega$ in $\mathcal{R}\left(\mathbb{R}^{n}\right), C^{3}(\partial \Omega)$ is a Baire space, it follows from our definition of the topology on $\mathcal{R}\left(\mathbb{R}^{n}\right)$ that $\mathcal{R}\left(\mathbb{R}^{n}\right)$ is also a Baire space. (Proceeding as in [7, 4.4.7], one can also prove that $\mathcal{R}\left(\mathbb{R}^{n}\right)$ with our topology is a $C^{0}$-manifold modeled on the Banach spaces $C^{3}(\partial \Omega)$ with $\Omega \in \mathcal{R}\left(\mathbb{R}^{n}\right)$. But we do not need that property.)

Let us recall that that a property $(P)$ holds for generic $\Omega \in \mathcal{R}\left(\mathbb{R}^{n}\right)$ if there exists a residual subset $\tilde{D} \subset \mathcal{R}\left(\mathbb{R}^{n}\right)$ such that property $(\mathrm{P})$ holds for every $\Omega \in \tilde{D}$.

Theorem 2. Condition (A) holds for generic $\Omega \in \mathcal{R}\left(\mathbb{R}^{n}\right)$.

Proof. The strategy of proof is standard and goes as follows (cf. [1]). Let $\mathcal{G} \subset \mathcal{R}\left(\mathbb{R}^{n}\right)$ be the set of $\Omega \in \mathcal{R}\left(\mathbb{R}^{n}\right)$ such that

(a) all eigenvalues of $-\Delta_{\Omega}^{D}$ are simple,

(b) $\int_{\Omega} w d x \neq 0$, for every non zero eigenfunction $w$ of $-\Delta_{\Omega}^{D}$.

Similarly, for every positive integer $l$, the set $\mathcal{S}_{l} \subset \mathcal{R}\left(\mathbb{R}^{n}\right)$ (respectively $\mathcal{G}_{l} \subset \mathcal{R}\left(\mathbb{R}^{n}\right)$ ) of open sets $\Omega \in \mathcal{R}\left(\mathbb{R}^{n}\right)$ is defined such that property (a) (respectively, and property $(b)$ ) holds at least for the first $l$ eigenvalues of $-\Delta_{\Omega}^{D}$. Clearly, $\mathcal{G}$ is the countable intersection of the $\mathcal{G}_{l}$ 's.

We show next that $\mathcal{G}$ is residual, which implies Theorem 2 Indeed, if property $(a)$ holds for $-\Delta_{\Omega}^{D}$, then, by applying the Weyl formula for $-\Delta_{\Omega}^{D}$ (cf. [21, Theorem 15.2, p.124]), one deduces that $\lambda_{k} \sim_{k \rightarrow \infty} C(\Omega) k^{2 / n}$, where $0<\lambda_{1}<\lambda_{2}<\cdots<\lambda_{j}<\lambda_{j+1}<\cdots$ is the ordered sequence of the eigenvalues of the Laplace-Dirichlet operator $-\Delta_{\Omega}^{D}$. Therefore, property $(A)$ holds.

For $l \geqslant 0, \mathcal{S}_{0}=\mathcal{G}_{0}:=\mathcal{R}\left(\mathbb{R}^{n}\right), \mathcal{G}_{l} \subset \mathcal{S}_{l}, \mathcal{S}_{l+1} \subset \mathcal{S}_{l}$ and $\mathcal{S}:=\cap_{l \geqslant 0} \mathcal{S}_{l}$ and, similarly, $\mathcal{G}_{l+1} \subset \mathcal{G}_{l}$ and $\mathcal{G}=\cap_{l \geqslant 0} \mathcal{G}_{l}$. Moreover, for $l \geqslant 0$, it is clear that the sets $\mathcal{S}_{l}$ and $\mathcal{G}_{l}$ are open in $\mathcal{R}\left(\mathbb{R}^{n}\right)$ (see [1]). To show that $\mathcal{G}$ is residual, amounts to establish the next lemma.

Lemma 2. For every $l \geqslant 0, \mathcal{G}_{l+1}$ is dense in $\mathcal{G}_{l}$. 


\section{Proof of Lemma 2 .}

First, recall that, for every $l \geqslant 0, S_{l}$ is dense in $\mathcal{R}\left(\mathbb{R}^{n}\right)$ (see [24]).

We follow the lines of the argument of Theorem 2 in [1]]. Let $\Omega \in \mathcal{G}_{l}$. It is sufficient to exhibit $\Omega^{\prime} \in \mathcal{G}_{l+1}$, arbitrarily close to $\Omega$. Since $\mathcal{S}_{l+1}$ is dense, it is enough to establish the previous fact for $\Omega \in \mathcal{G}_{l} \cap \mathcal{S}_{l+1}$. Let $\left(\mu_{k}\right)_{k \in \mathbb{N}^{*}}$ be the ordered sequence of the eigenvalues of the Laplace-Dirichlet operator $-\Delta_{\Omega}^{D}$ repeated according to their multiplicity. We have

$$
\mu_{1}<\mu_{2}<\cdots \mu_{l}<\mu_{l+1}<\mu_{l+2} \leqslant \mu_{l+3} \leqslant \cdots .
$$

Let $w_{l+1}$ be an eigenfunction of $-\Delta_{\Omega}^{D}$ for the eigenvalue $\mu_{l+1}$. If $\int_{\Omega} w_{l+1} d x \neq 0$, then $\Omega \in \mathcal{G}_{l+1}$. Otherwise, we may assume that

$$
\int_{\Omega} w_{l+1} d x=0
$$

and we simply use $\mu$ and $w$ to denote $\mu_{l+1}$ and $w_{l+1}$. Let $\xi \in C^{3}\left(\partial \Omega ; \mathbb{R}^{n}\right)$ be such that 2.12) holds and let $\varepsilon_{0}>0$ be as above (see (i) and (ii) in this subsection). Set $\varepsilon_{0}^{\prime}$ as the minimum of $\xi(\pi(x)) \cdot(\pi(x)-x) ; x \in \Omega$ with $\operatorname{dist}(x, \partial \Omega)=\varepsilon_{0} / 2$ is positive. Let $\rho \in C^{\infty}(\mathbb{R},[0,1])$ be such that

$$
\begin{aligned}
& \rho=1 \text { on a neighborhood of }(-\infty, 0], \\
& \rho=0 \text { on a neighborhood of }\left[\varepsilon_{0}^{\prime},+\infty\right) .
\end{aligned}
$$

We use $C_{\varepsilon}^{3}(\partial \Omega)$ to denote the set of $\eta \in C^{3}(\partial \Omega)$ such that $|\eta|_{C^{3}(\partial \Omega)}<\varepsilon$. For $\eta \in C_{\varepsilon}^{3}(\partial \Omega)$, we consider $h_{\eta}: \bar{\Omega} \rightarrow \mathbb{R}^{n}$ defined by

$$
h_{\eta}(x):=x
$$

for every $x \in \Omega$ with $\operatorname{dist}(x, \partial \Omega) \geqslant \varepsilon_{0} / 2$ and

$$
h_{\eta}(x):=x+\eta(\pi(x))
$$

$$
\left(1-\rho\left(\varepsilon_{0}^{\prime}-\xi(\pi(x)) \cdot(\pi(x)-x)\right)\right) \xi(\pi(x)),
$$

for every $x \in \bar{\Omega}$ with $\operatorname{dist}(x, \partial \Omega) \leqslant \varepsilon_{0} / 2$. We now fix $\varepsilon \in\left(0, \varepsilon_{0}\right)$ small enough so that, for every $\eta \in C_{\varepsilon}^{3}(\partial \Omega), h_{\eta}$ is a diffeomorphism of class $C^{3}$ from $\bar{\Omega}$ into $\overline{\Omega_{\eta}}$. Let $P: H^{2}(\Omega) \rightarrow H^{2}\left(\mathbb{R}^{n}\right)$ be a linear continuous map such that

$$
P(v)=v \text { in } \Omega \text {. }
$$

For $\eta \in C_{\varepsilon}^{3}(\partial \Omega)$, let $Q_{\eta}: H^{2}\left(\mathbb{R}^{n}\right) \rightarrow H_{0}^{1}\left(\Omega_{\eta}\right) \cap H^{2}\left(\Omega_{\eta}\right), \phi \mapsto \psi$, be defined by

$$
\begin{gathered}
-\Delta \psi=-\Delta \phi \text { in } L^{2}\left(\Omega_{\eta}\right), \\
\psi=0 \text { on } \partial \Omega_{\eta} .
\end{gathered}
$$

Consider the set $E$ of pairs $(v, \eta) \in H^{2}(\Omega) \times C_{\varepsilon}^{3}(\partial \Omega)$ with $v(x)+\eta(x) \frac{\partial w}{\partial \xi}(x)=0$ for every $x \in \partial \Omega$, and the following map $\Phi: E \times \mathbb{R} \rightarrow L^{2}(\Omega) \times \mathbb{R}$ which associates to $((v, \eta), \chi)$,

$$
\left(\left((-\Delta-\chi)\left(Q_{\eta}(P(v))\right)\right) \circ h_{\eta}, \int_{\Omega_{\eta}} Q_{\eta}(P(v)) d x\right) .
$$


One has $\Phi((w, 0), \mu)=(0,0)$ and Lemma 2 holds if $\Phi$ is locally onto at $((w, 0), \mu)$. The map $\Phi$ is of class $C^{1}$ and one has

$$
\Phi^{\prime}((w, 0), \mu)((v, \eta), \chi)=\left(-\Delta v-\mu v-\chi w, \int_{\Omega} v d x\right),
$$

for every $(v, \eta) \in H^{2}(\Omega) \times C^{3}(\partial \Omega)$ such that

$$
v(x)+\eta(x) \frac{\partial w}{\partial \xi}(x)=0, \forall x \in \partial \Omega .
$$

Using the Fredholm alternative (recall that the eigenvalue $\mu$ is assumed to be simple), one easily checks that, for every $f \in L^{2}(\Omega)$ and every $\eta \in C^{3}(\partial \Omega)$, there exists one and only one $(v, \chi) \in$ $H^{2}(\Omega) \times \mathbb{R}$ such that

$$
\begin{gathered}
-\Delta v-\mu v-\chi w=f, \\
\int_{\Omega} v w d x=0, \\
v(x)+\eta(x) \frac{\partial w}{\partial \xi}(x)=0, \forall x \in \partial \Omega .
\end{gathered}
$$

For $f=0$, let us denote by $\left(v_{\eta}, \chi_{\eta}\right)$ the corresponding unique solution. We next prove that

$$
\text { there exists } \eta_{0} \in C^{3}(\partial \Omega) \text { such that } \int_{\Omega} v_{\eta_{0}} d x \neq 0 \text {. }
$$

To compute $\int_{\Omega} v_{\eta} d x$ in terms of $\eta$, we consider the unique solution to the inhomogeneous Dirichlet problem given by

$$
\begin{cases}(-\Delta-\mu) S=1, & \text { in } \Omega, \\ S=0, & \text { on } \partial \Omega, \\ \int_{\Omega} S w d x=0 . & \end{cases}
$$

Since $\int_{\Omega} w d x=0$ and the eigenvalue $\mu$ is simple, the Fredholm alternative tells us that such an $S$ exists (and is unique). By applying Stokes' formula, one gets, using in particular (2.15), (2.16), (2.17) and (2.19),

$$
\int_{\Omega} v_{\eta} d x=\int_{\Omega}((-\Delta-\mu) S) v_{\eta} d x=\int_{\partial \Omega} \eta \frac{\partial S}{\partial v} \frac{\partial w}{\partial v} d \sigma .
$$

Let us assume that (2.18) does not hold. Then, the right hand side of (2.20) should be equal to zero for every $\eta \in C^{3}(\partial \Omega)$ and, therefore,

$$
\frac{\partial S}{\partial v} \frac{\partial w}{\partial v} \equiv 0
$$

By the Holmgren uniqueness theorem (see e.g. [23, Proposition 4.3, p. 433]), since $w$ is a non zero eigenfunction of $-\Delta_{\Omega}^{D}, \partial w / \partial v$ cannot be equal to zero on any nonempty open subset of $\partial \Omega$. Therefore, for the previous equation to hold, it results that

$$
\frac{\partial S}{\partial \nu}=0 \text { on } \partial \Omega .
$$

The following lemma tells us that (2.21) cannot hold true (and, therefore, yields (2.18). 
Lemma 3. With the notations above, there is no solution to the following over determined eigenvalue problem

$$
\begin{cases}(-\Delta-\mu) S=1, & \text { in } \Omega, \\ S=0, & \text { on } \partial \Omega, \\ \frac{\partial S}{\partial v}=0, & \text { on } \partial \Omega .\end{cases}
$$

The result is classical and we will not provide here a proof. Then, it is then easy to conclude the argument for Theorem 2 .

\section{Steady-state controllability for a water tank}

Let us consider the controllability problem for a tank containing a fluid. As in [19], we consider an open, bounded and connected subset $\Omega$ of $\mathbb{R}^{2}$, which corresponds to the shape of the tank. The mathematical description of this problem is given by the position $D$ in $\mathbb{R}^{2}$ of the tank and by the height $h(t, x)$ of the fluid with respect to an equilibrium position. The control system is modeled by

$$
\begin{cases}\ddot{D}(t)=u(t), & \text { if } t \in(0, T), \\ h_{t t}(t, x)=\Delta h(t, x), & \text { if }(t, x) \in(0, T) \times \Omega, \\ \frac{\partial h}{\partial v}(t, x)=-u(t) \cdot v(x), & \text { if }(t, x) \in(0, T) \times \partial \Omega\end{cases}
$$

where the control $u(t) \in \mathbb{R}^{2}$. Here $v(x)$ denotes again the outward unit normal vector at $x \in \partial \Omega$. The steady-state control problem is the following one. Let $D_{0}$ and $D_{1}$ be two arbitrary points in $\mathbb{R}^{2}$, does there exist $T>0$ and $u:[0, T] \rightarrow \mathbb{R}^{2}$ such that the solution $D:[0, T] \rightarrow \mathbb{R}^{2}, h:[0, T] \times \Omega \rightarrow \mathbb{R}$ of (3.1) with

$$
h(0, \cdot)=0, h_{t}(0, \cdot)=0, D(0)=D_{0}, \dot{D}(0)=0
$$

satisfies

$$
D(T)=D_{1}, \dot{D}(T)=0, h(T, \cdot)=h_{t}(T, \cdot)=0 ?
$$

In [19], N. Petit and P. Rouchon proved that, if the shape $\Omega$ of the tank is a rectangle or a circle, then there is a solution to this controllability problem. When $\Omega$ has a general form, they assert the problem is open. Here, in the spirit of the first part of this paper, we propose a necessary condition for that steady-state controllability concerning the behavior of eigenvalues and eigenfunctions of a Neumann problem.

Let us fix $\Omega \subseteq \mathbb{R}^{2}$ a bounded, open and connected subset of $\mathbb{R}^{2}$ of class $C^{2}$ or a convex polygon. Let us first recall some classical results about the weak solution to the following Cauchy problem

$$
\begin{cases}\ddot{D}(t)=u(t), & \text { if } t \in(0, T), \\ \dot{D}(0)=s_{0}, & \\ D(0)=D_{0}, & \text { if }(t, x) \in(0, T) \times \Omega, \\ h_{t t}(t, x)=\Delta h(t, x), & \text { if }(t, x) \in(0, T) \times \partial \Omega, \\ \frac{\partial h}{\partial v}(t, x)=-u(t) \cdot v(x), & \text { if } x \in \Omega, \\ h(0, x)=h_{0}(x), & \text { if } x \in \Omega . \\ h_{t}(0, x)=v_{0}(x), & \end{cases}
$$


Define

$$
\begin{aligned}
& H:=\left\{h \in L^{2}(\Omega) ; \int_{\Omega} h d x=0\right\}, \\
& V:=\left\{h \in H^{1}(\Omega) ; \int_{\Omega} h d x=0\right\},
\end{aligned}
$$

and let $V^{\prime}$ be the dual space of $V \subset H$. Let $D_{0} \in \mathbb{R}^{2}, s_{0} \in \mathbb{R}^{2},\left(h_{0}, v_{0}\right) \in H \times V^{\prime}, T>0$ and $u \in L^{2}\left(0, T ; \mathbb{R}^{2}\right)$. A weak solution to the Cauchy problem (3.4) is a couple $(D, h)$ such that

$$
\begin{gathered}
D \in H^{2}\left(0, T ; \mathbb{R}^{2}\right), D(0)=D_{0}, \\
\dot{D}(0)=s_{0}, \ddot{D}=u \in L^{2}(0, T), \\
h \in C^{0}([0, T] ; H) \cap C^{1}\left([0, T] ; V^{\prime}\right),
\end{gathered}
$$

and such that, for every $\tau \in[0, T]$ and for every

$$
\theta \in C^{0}\left([0, T] ; H^{2}(\Omega)\right) \cap C^{1}\left([0, T] ; H^{1}(\Omega)\right) \cap C^{2}\left([0, T] ; L^{2}(\Omega)\right)
$$

satisfying

$$
\begin{gathered}
\theta_{t t}=\Delta \theta, \text { in } C^{0}\left([0, T] ; L^{2}(\Omega)\right), \\
\frac{\partial \theta}{\partial v}=0, \text { in } C^{0}\left([0, T] ; H^{1 / 2}(\partial \Omega)\right),
\end{gathered}
$$

one has

$$
\begin{aligned}
&-\int_{0}^{\tau} \int_{\partial \Omega} \theta(t, x) u(t) \cdot v(x) d \sigma(x) d t+\left\langle v_{0}, \theta(0, \cdot)\right\rangle_{V^{\prime}, V} \\
&-\int_{\Omega} h_{0}(x) \theta_{t}(0, x) d x=\left\langle h_{t}(\tau, \cdot), \theta(\tau, \cdot)\right\rangle_{V^{\prime}, V} \\
& \quad-\int_{\Omega} h(\tau, x) \theta_{t}(\tau, x) d x .
\end{aligned}
$$

Of course, for every $D \in H^{2}(0, T)$ and every $h \in C^{0}\left([0, T] ; H^{2}(\Omega)\right) \cap C^{1}\left([0, T] ; H^{1}(\Omega)\right) \cap$ $C^{2}\left([0, T] ; L^{2}(\Omega)\right)$, if $(D, h)$ is a classical solution to (3.4), then it is also a weak solution to (3.4). Moreover, it is well known that, for every $\left(D_{0}, s_{0}\right) \in \mathbb{R}^{2} \times \mathbb{R}^{2},\left(h_{0}, v_{0}\right) \in H \times V^{\prime}, T>0$ and $u \in L^{2}\left(0, T ; \mathbb{R}^{2}\right)$, there exists one and only one weak solution $(D, h)$ to (3.4). This unique $(D, h)$ is called the solution to the Cauchy problem (3.4).

We say that the control system (3.1) is steady-state controllable if, for every $\left(D_{0}, D_{1}\right) \in \mathbb{R}^{2} \times$ $\mathbb{R}^{2}$, there exist $T>0$ and $u \in L^{2}\left(0, T ; \mathbb{R}^{2}\right)$ with $u(0)=0$ such that the solution to the Cauchy problem (3.4), with $h_{0}=v_{0}=0, s_{0}=0$, satisfies (3.3).

Consider the Laplace-Neumann operator $-\Delta_{\Omega}^{N}$ defined as follows:

$$
\begin{gathered}
\mathcal{D}\left(-\Delta_{\Omega}^{N}\right):=\left\{v \in H^{2}(\Omega) ; \frac{\partial v}{\partial v}=0 \text { on } \partial \Omega\right\}, \\
-\Delta_{\Omega}^{N} v=-\Delta v, \forall v \in \mathcal{D}\left(-\Delta_{\Omega}^{N}\right) .
\end{gathered}
$$




\subsection{A condition that prevents steady-state controllability}

We next introduce property $(B)$ which turns out to prevent steady-state controllability in finite time.

Definition 2. The open set $\Omega$ has the property $(B)$ if there exists a sequence $\left(\lambda_{k}\right)_{k \in \mathbb{N}^{*}}$ of distinct eigenvalues of $-\Delta_{\Omega}^{N}$ such that

(i) there exist $\rho \in(0,2)$ and $C>0$ such that

$$
\lambda_{k} \leqslant C k^{\rho}, \forall k \geqslant 1
$$

(ii) for every $k \in \mathbb{N}^{*}$, there exists an eigenfunction $w_{k}$ for the eigenvalue $\lambda_{k}$ and the operator $-\Delta_{\Omega}^{N}$ such that

$$
\int_{\partial \Omega} w_{k} v d \sigma \neq 0
$$

We are now able to state the following result.

Theorem 3. If $\Omega$ has property (B), then the control system (3.1) is not steady-state controllable.

The proof is similar to that of Theorem 1 .

\subsection{Genericity of condition $(B)$}

In this section, we prove that condition $(B)$ holds generically for tank shapes of class $C^{3}$, and therefore by Theorem 3 , for such generic tank shapes $\Omega$, steady-state controllability for a watertank does not hold.

We use here notations and results of [22]. Let $S_{3}$ be the set of all non empty open, bounded, connected subsets $\Omega \subset \mathbb{R}^{2}$ of class $C^{3}$. The topology on $\mathcal{S}_{3}$ is defined as follows ([22, p. 7]).

Let $C_{b}^{3}\left(\mathbb{R}^{2}, \mathbb{R}^{2}\right)$ be the space of functions $u: \mathbb{R}^{2} \rightarrow \mathbb{R}^{2}$ of class $C^{3}$ such that

$$
\begin{gathered}
\|u\|_{3}:=\operatorname{Sup}\left\{\left|\partial^{\alpha} u(x)\right| ; x \in \mathbb{R}^{2}, \alpha=\left(\alpha_{1}, \alpha_{2}\right) \in \mathbb{N}^{2}\right. \\
\text { with } \left.\alpha_{1}+\alpha_{2} \leqslant 3\right\}<+\infty .
\end{gathered}
$$

Then $C_{b}^{3}\left(\mathbb{R}^{2}, \mathbb{R}^{2}\right)$ equipped with the norm $\|\cdot\|_{3}$ is a Banach space. For $\Omega \in S_{3}$ and $u \in C_{b}^{3}\left(\mathbb{R}^{2}, \mathbb{R}^{2}\right)$, let $\Omega+u:=(I d+u)(\Omega)$ be the subset of points $y \in \mathbb{R}^{2}$ such that $y=x+u(x)$ for some $x \in \Omega$. By simple topological arguments, one easily gets that, for $u \in C_{b}^{3}\left(\mathbb{R}^{2}, \mathbb{R}^{2}\right)$ small enough, $\Omega+u$ belongs to $\mathcal{S}_{3}$.

For $\varepsilon>0$, let $\mathcal{V}(\varepsilon)$ be the sets of all the $\Omega+u$ with $u \in C_{b}^{3}\left(\mathbb{R}^{2}, \mathbb{R}^{2}\right)$ and $\|u\|_{3}<\varepsilon$. The topology on $S_{3}$ is defined by considering the sets $\mathcal{V}(\varepsilon)$ with $\varepsilon$ small enough as a base of neighborhoods of $\Omega$. Then $\mathcal{S}_{3}$ is a Baire space.

Theorem 4. Condition (B) holds for generic $\Omega \in \mathcal{S}_{3}$.

The proof follows the lines of that of Theorem 2 but is much more involved. 


\section{References}

[1] J. H. Albert, Genericity of simple eigenvalues for elliptic PDE's, Proc. Amer. Math. Soc. 48 (1975), pp. 413-418.

[2] S. A. Avdonin, S. A. Ivanov, Families of exponentials, Cambridge University Press, 1995.

[3] J.-M. Coron, Local controllability of a 1-D tank containing a fluid modeled by the shallow water equations, ESAIM: COCV, 8 (2002), pp. 513-554.

[4] F. Dubois, N. Petit and P. Rouchon, Motion planning and nonlinear simulations for a tank containing a fluid, ECC 99.

[5] D. Bresch, J. Simon, On the normal variations of a domain, ESAIM: COCV 3 (1998), pp. 251-261.

[6] H. O. Fattorini, Control in finite time of differential equations in Banach space, Comm. Pure Appl. Math. 19 (1966), pp. 17-34.

[7] R. S. Hamilton, The inverse function theorem of Nash and Moser, Bull. Amer. Math. Soc. (N.S.) 7 (1982), pp. 65-222.

[8] V. Ivrii, Microlocal analysis and precise spectral asymptotics, SMM, Springer, 1991.

[9] J. C. Lagarias, A. M. Odlyzko, Effective versions of the Chebotarev density theorem, Algebraic number fields: $L$-functions and Galois properties (Proc. Sympos., Univ. Durham, Durham, 1975), pp. 409-464. Academic Press, London, 1977.

[10] B. Ya Levin, Lectures on entire functions, Translations of mathematical monographs, 150, American mathematical society, Rhode Island, USA, 1996.

[11] J.-L. Lions, E. Zuazua, Approximate controllability of a hydro-elastic coupled system, ESAIM: COCV 1 (1996), pp. 1-15.

[12] F. Murat, J. Simon, Sur le contrôle par un domaine géométrique, Rapport du L.A. 189, 76015, Université Paris VI, 1976.

[13] S. Micu, E. Zuazua, On the lack of null controllability of the heat equation on the half line, Transactions of the A.M.S. 353 (2001), pp. 1635-1659.

[14] S. Micu, E. Zuazua, On the lack of null controllability of the heat equation on the half space, Portugaliae Mathematica 58 (2001), pp. 1-24.

[15] S. Micu, E. Zuazua, On the controllability of a fractional order parabolic equation, preprint, Universidad Autónoma de Madrid, 2004.

[16] J. H. Ortega, E. Zuazua, Generic simplicity of the spectrum and stabilization for a plate equation, SIAM J. Control Optim. 39 (2000), pp. 1585-1614.

[17] J. H. Ortega, E. Zuazua, Generic simplicity of the eigenvalues of the Stokes system in two space dimensions, Adv. Differential Equations 6 (2001), pp. 987-1023.

[18] J. H. Ortega, E. Zuazua, On a constrained approximate controllability problem for the heat equation, Technical Note, J. of Optm. Theory and App. 1 (2003), pp. 183-190.

[19] N. Petit, P. Rouchon, Dynamics and solutions to some control problems for water tank systems, IEEE Trans. Automat. Control 47 (2002), pp. 594-609.

[20] P. Rouchon, Calcul symbolique et génération de trajectoires pour certaines EDP avec contrôle frontière, lecture at the Collège de France, Décembre 2003. 
[21] M. A. Shubin, Pseudodifferential operators and spectral theory, Springer Series in Soviet Math., Springer-Verlag, (1987).

[22] J. Simon, Differenciacion de problemas de contorno respecto del dominio, Universidad de Sevilla (1991) 122 pages.

[23] M. E. Taylor, Partial differential equations I, Applied mathematical sciences, vol. 115, Springer-Verlag, New York, 1996.

[24] K. Uhlenbeck, Generic properties of eigenfunctions, Amer. J. Math. 98 (1976), pp. 1059-1078. 